\title{
Self-consistent intermediate Hamiltonians: A coupled cluster type formulation of the singles and doubles configuration interaction matrix dressing
}

\author{
I. Nebot-Gil and J. Sánchez-Marín \\ Departament de Química Física, Facultat de Química, Universitat de València, c/Dr. Moliner, \\ 5046100 Burjassot (Valencia), Spain \\ J. P. Malrieu, J. L. Heully, and D. Maynau \\ Laboratoire de Physique Quantique, URA 505 du CNRS, Université Paul Sabatier, 118, Route de Narbonne, \\ 31062 Toulouse Cedex, France
}

(Received 27 January 1995; accepted 27 April 1995)

\begin{abstract}
This paper presents a new self-consistent dressing of a singles and doubles configuration interaction matrix which insures size-consistency, separability into closed-shell subsystems if localized molecular orbitals (MOs) are used, and which includes all fourth order corrections. This method yields, among several schemes, a reformulation of the coupled cluster method, including fully the cluster operators of single and double excitations, and partially those of the triples (Bartlett's algorithm named CCSDT-1a). Further improvement can be easily included by adding exclusion principle violating corrections. Since it leads to a matrix diagonalization, the method behaves correctly in case of near degeneracies between the reference determinant and some doubles. Due to its flexibility this formulation offers the possibility of consistent combination with less expensive treatments for the study of very large systems. () 1995 American Institute of Physics.
\end{abstract}

\section{INTRODUCTION}

The size-consistency requirement is a fundamental aspect of the quantum many-body problem. ${ }^{1-4}$ While truncated configuration interactions (CI) are variational and suitable for rational and flexible selections, they are not size-consistent. ${ }^{5}$ A more correct scheme is the coupled cluster $(\mathrm{CC})^{6-12}$ expansion. At its singles and doubles approximation (CC-SD), it ignores part of the triples and thus does not insure the Rayleigh-Schrödinger fourth order. The complete inclusion of the triples is rather expensive, and thus perturbative treatments of the triples are frequently proposed $(\operatorname{CCSD}(\mathrm{T})),{ }^{13-15}$ although they do not behave properly when single bonds are broken. Although the full CCSDT model has been developed ${ }^{16-18}$ the calculations performed keep rather illustrative character due to the cost of the method. Moreover, since the $\mathrm{CC}$ equations are nonlinear, they must be solved iteratively. The numerical algorithms for solving sets of nonlinear equations suffer for risk of poor convergence and require rather large number of iterations. ${ }^{19,20}$

Somewhere in between $\mathrm{CI}$ and $\mathrm{CC}$ one must mention the approximate size-extensive CEPA (coupled electron pair

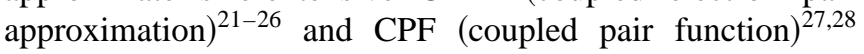
models, which exist in several versions and are essentially based on a single reference and truncation to double excitations, plus an approximate cancellation of the unlinked corrections.

In a previous work, ${ }^{29}$ the size extensivity of the lowest root of any selected CI has been obtained by a proper dressing (or change) of the diagonal energies of the CI matrix. This dressing is self-consistent, depending on the coefficients of the doubles, so that the method has been labeled $(\mathrm{SC})^{2} \mathrm{CI}$ (size-consistent self-consistent CI). Its implementation is straightforward with negligible extra costs in terms of memory and computation time, and the efficiency is really impressing ${ }^{30,31}$ specially when a direct selected CI algorithm is used. ${ }^{32}$

The method was derived in terms of the intermediate Hamiltonian Theory ${ }^{33}$ (a generalization of effective Hamiltonians ${ }^{33}$ ), but it may be seen as the most exact CEPA scheme when the model space is the SDCI space or as a generalized CEPA scheme for arbitrary CI's. It insures the strict separability of the energy

$$
E_{A B} \rightarrow E_{A}+E_{B} \quad \text { when } r_{A B} \rightarrow \infty
$$

for the separation of an $A B$ system into $A$ and $B$ closed shell subsystems provided that localized MO's are used. The method consists in adding unlinked effects of the outer space onto the diagonal energies in order to cancel all unlinked terms produced by the diagonalization. Of course, if the model space consists in the SDCI space, the linked contributions of triples and quadruples are not taken into account so that the method is poorer than CC-SD which correctly treats the linked contribution of the quadruples.

The linked effects of the triples and quadruples have been added once ${ }^{35}$ a posteriori as the mean value of an additional dressing operator, taken on the vector resulting from the $(\mathrm{SC})^{2} \mathrm{SDCI}$. Actually, this was an approximate application of a fundamental idea, namely the total dressing of the model space by the linked and unlinked effects of the outer space.

The preceding paper ${ }^{36}$ has presented a simple definition of a diagonal dressing in terms of the coefficients of the desired vector on the outer space determinants, together with two perturbative evaluations of these coefficients (and therefore of the dressing). The test calculations were convincing, especially when high-order exclusion principle violating (EPV) corrections were included. The present paper explores the possibility to replace these perturbative evaluations of the 
dressing by coupled cluster type estimates, which are expected to behave more satisfactorily when near degeneracies occur in the model space. A formulation is found, which is equivalent to CCSD method, if only the unlinked contributions to triples and quadruples are taken into account, or analogous to CCSDT-1a, ${ }^{37,38}$ if the linked contributions to Triples are perturbatively accounted for. Therefore, the method insures the strict separability into closed shell subsystems when localized MO's are used. Although leading to a matrix built on the SDCI space, the method treats all the fourth order effects of the triples and quadruples.

\section{SELF-CONSISTENT INTERMEDIATE HAMILTONIAN WITH HERMITIAN DRESSINGS}

\section{A. Matrix formulation of the intermediate Hamiltonians}

We shall not recall the definition and advantage of intermediate Hamiltonians. ${ }^{33}$ We suppose that we only search the exact energy and the projection of the exact eigenvector onto the model space of a single root, this eigenvector having the largest amplitude on the main model space determinant $\Phi_{0}$ (or reference determinant). The intermediate model space is spanned by (at least) all the determinants $\Phi_{i}$ interacting with $\Phi_{0}$. Thus, $\Phi_{0}$ and all the $\Phi_{i}$ built the model space $S$. Then, if $P$ is the projector on the model space $S$

$$
P=\left|\Phi_{0}\right\rangle\left\langle\Phi_{0}\left|+\sum_{i \in S}\right| \Phi_{i}\right\rangle\left\langle\Phi_{i}\right|,
$$

it leads to the diagonalization of the dressed matrix

$$
\mathbf{P}(\mathbf{H}+\mathbf{\Delta}) \mathbf{P},
$$

where $\Delta$ is the dressing operator.

If we define $\mathbf{Q}=\mathbf{1}-\mathbf{P}$ as the projection on the outer space, and $\mathbf{c}$ as the vector of the coefficients of the exact eigenvector:

$$
\Psi_{0}=\Phi_{0}+\sum_{i \in S} c_{i} \Phi_{i}+\sum_{\alpha \notin S} c_{\alpha} \Phi_{\alpha}
$$

the exact Schrödinger equation $\left(\mathscr{H}-\mathscr{E}_{0}\right) \Psi_{0}=0$ may be written for the rows associated to the model space in a matricial formulation:

$$
\mathbf{P H P c}-\mathscr{E}_{0} \mathbf{P 1 P c}+\mathbf{P H Q c}=0 .
$$

The last term PHQc is a vector $\mathbf{V}$ the elements of which are

$$
V_{i}=\sum_{\alpha \notin S} c_{\alpha}\left\langle\Phi_{i}|\mathscr{H}| \Phi_{\alpha}\right\rangle
$$

so that Eq. (5) may be written

$$
\mathbf{P H P c}-\mathscr{E}_{0} \mathbf{P} 1 \mathrm{Pc}=-\mathbf{V} .
$$

The dressed matrix eigenequation is

$$
\left[\mathbf{P}(\mathbf{H}+\boldsymbol{\Delta}) \mathbf{P}-\mathscr{E}_{0} \mathbf{P} 1 \mathbf{P}\right] \mathbf{P c}=0 .
$$

The condition under which the above equation will provide the exact energy and the exact components of the eigenvector on the model space is that

$$
\mathbf{V}=\mathbf{P} \boldsymbol{\Delta} \mathbf{P c}=\boldsymbol{\Delta} \mathbf{P c}
$$

or simply, restricted to the model space:

$$
\mathbf{V}=\boldsymbol{\Delta} \mathbf{c} \text {. }
$$

This equation is not sufficient to define $\boldsymbol{\Delta}$. Of course, $\Delta$ might be a full square matrix as occurs in the partitioning technique. ${ }^{39-42}$ However, it is sufficient to define the dressing through $n$ matrix elements only since there are only $n$ degrees of freedom ( 1 eigenenergy, $n-1$ coefficients).

\section{B. Dressing formulations}

One possibility is the diagonal dressing ${ }^{43}$

$$
\begin{aligned}
& \Delta_{i j}=\Delta_{i i} \delta_{i j}, \\
& \Delta_{i i}=c_{i}^{-1} \sum_{\alpha \notin S} c_{\alpha}\left\langle\Phi_{i}|\mathscr{H}| \Phi_{\alpha}\right\rangle .
\end{aligned}
$$

This solution has the drawback of the division by $c_{i}$ which may lead to numerical instabilities and the possible appearance of physically meaningless eigenenergies of $\mathbf{P}(\mathbf{H}+\boldsymbol{\Delta}) \mathbf{P}$ below the relevant root $\mathscr{E}_{0}$.

Two of us have proposed elsewhere a first column dressing, ${ }^{43}$ concerning the elements $\left\langle\Phi_{i}|\Delta| \Phi_{0}\right\rangle$

$$
\left\langle\Phi_{i}|\Delta| \Phi_{0}\right\rangle=\sum_{\alpha \notin S} c_{\alpha}\left\langle\Phi_{i}|\mathscr{H}| \Phi_{\alpha}\right\rangle
$$

which does not lead to such numerical troubles but which is non-hermitian.

We strongly recommend a new formulation of the dressing concerning the first column and first row only. The first column of $\boldsymbol{\Delta}$ is identical to the preceding except for $\left\langle\Phi_{0}|\Delta| \Phi_{0}\right\rangle$, the dressing is made hermitian taking

$$
\left\langle\Phi_{0}|\Delta| \Phi_{i}\right\rangle=\left\langle\Phi_{i}|\Delta| \Phi_{0}\right\rangle
$$

and $\Delta_{00}$ is then calculated as

$\Delta_{00}=\left\langle\Phi_{0}|\Delta| \Phi_{0}\right\rangle=\sum_{\alpha \notin S} c_{\alpha}\left\langle\Phi_{0}|\mathscr{H}| \Phi_{\alpha}\right\rangle-\sum_{i \neq 0} c_{i}\left\langle\Phi_{i}|\Delta| \Phi_{0}\right\rangle$.

These relations are easily obtained from the matrix multiplication of the first row of $\boldsymbol{\Delta}$ by $\mathbf{c}$.

In the case where the model space includes all the determinants interacting with $\Phi_{0}$ the first summation is zero, and

$$
\Delta_{00}=-\sum_{i \neq 0} c_{i}\left\langle\Phi_{i}|\Delta| \Phi_{0}\right\rangle
$$

This formulation is hermitian and has shown to be numerically stable.

\section{Estimation of the coefficients of the outer space}

All these formulations remain academic as far as the coefficients on the outer space are unknown. Of course, in practice they can be approximately evaluated from the knowledge of the $c_{i}$ 's. Therefore these dressings require achieving a self-consistency condition. In the preceding paper $^{36}$ the coefficients $c_{\alpha}$ were evaluated perturbatively. This means that the coefficients for triple and quadruple excitations are obtained by a first order perturbation upon a vector built on all singly and doubly excited determinants. 


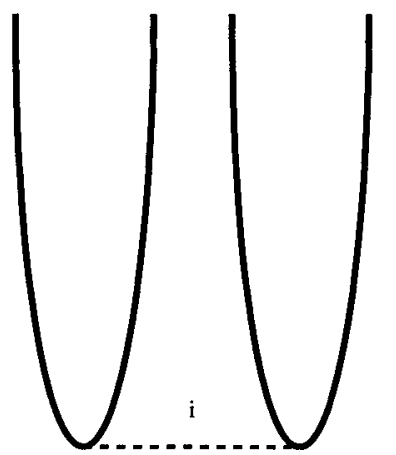

(a)

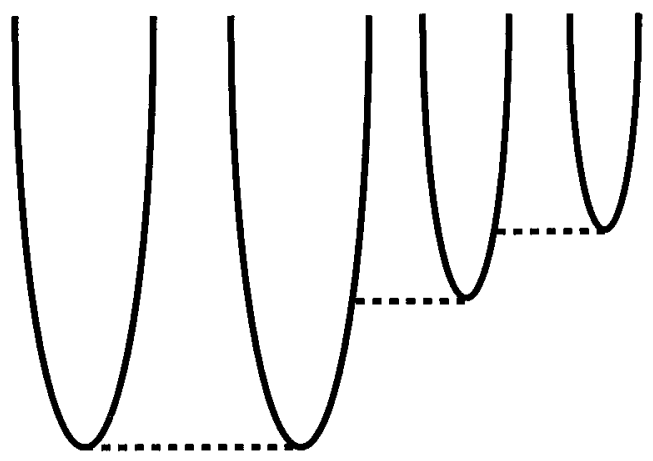

(b)

The use of these $c_{\alpha}$ 's in Eq. (8) gives an energy which is correct to fourth order and includes important fifth order terms. The present work will try to improve such a scheme by systematically using the factorization theorem which is part of perturbation theory. This will transform the preceding order-by-order expansion into a self-consistent, all-order approach.

The following paragraphs will show how such an allorder scheme is obtained, first for the quadruple excitations. Then the triple excitations will be considered. A comparison with the coupled cluster approach will follow. Finally, we shall show how further improvements can be obtained by considering the nonadditivity of the denominators (this additivity is implicit in the Möller-Plesset perturbation theory) or by including higher-order terms in the CEPA spirit, but this time for triple and quadruple excitations. As far as we know this is the first time that EPV terms are included for excitations higher than single and double ones.

\section{COUPLED CLUSTER FORMULATIONS OF SELF- CONSISTENT INTERMEDIATE HAMILTONIANS}

\section{A. Quadruple excitations}

Up to third order in the wave function, two kinds of quadruple excitations have to be considered. At the second order disconnected quadruples appear, the connected ones appearing at the next order (see Fig. 1).

Regarding the energy, the connected quadruple amplitudes will contribute for the first time at fifth order, and furthermore the coefficient $c_{\alpha}^{Q}$ cannot be written as a function of the $c_{i}$ 's. They have to be evaluated by perturbation, e.g., a
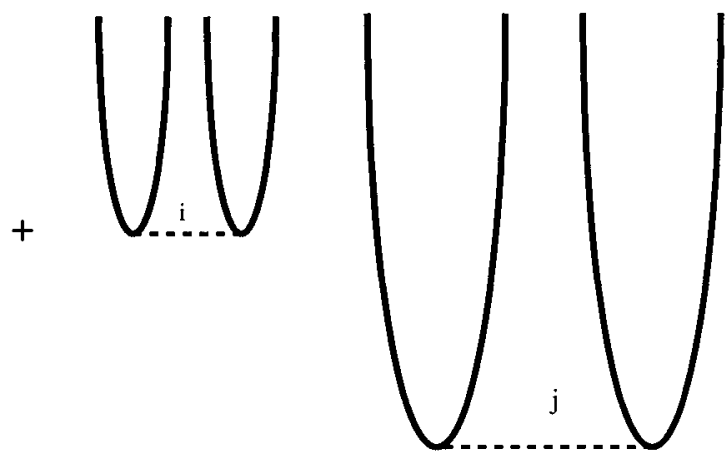

FIG. 1. (a) Disconnected and (b) connected contributions to the coefficients of the quadruples.

second order perturbation upon a singles and doubles vector. This is, of course, outside the scope of this method. The disconnected quadruples should be more important; their energy contribution appears at fourth order. By using the factorization theorem (see Ref. 44) one can show that the sum of the two terms of Fig. 1(a), can be rewritten as a product which by inspection yields the following second order relation

$$
c_{\alpha}^{Q}=\sum_{(i, j)} c_{i}^{(1)} c_{j}^{(1)}
$$

where $(i, j)$ stands for all couples of disjoint double excitations creating $\Phi_{\alpha}$ from $\Phi_{0}$

$$
\Phi_{\alpha}=D_{j}^{+} D_{i}^{+} \Phi_{0}=D_{k}^{+} D_{l}^{+} \Phi_{0}=\cdots .
$$

Equation (17), giving the coefficient of quadruples as products of double excitation coefficients, is generalized to an all-order relation by writing

$$
c_{\alpha}^{Q}=\sum_{\substack{(i, j) \\ D_{j}^{+} D_{i}^{+} \Phi_{0}=\Phi_{\alpha}}} c_{i} c_{j},
$$

where the $c_{i}$ coefficients are obtained by diagonalization of the dressed Hamiltonian, giving thus a self-consistent iterative method.

From this relation it is very easy to derive approximate schemes such as "exact CEPA method," called by us $(\mathrm{SC})^{2}$ MRCI and already presented in Ref. 29. These methods which considered EPV terms only will not be used here. 


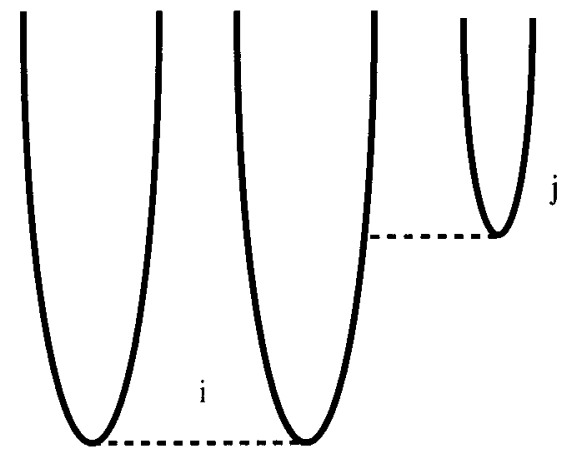

(a)
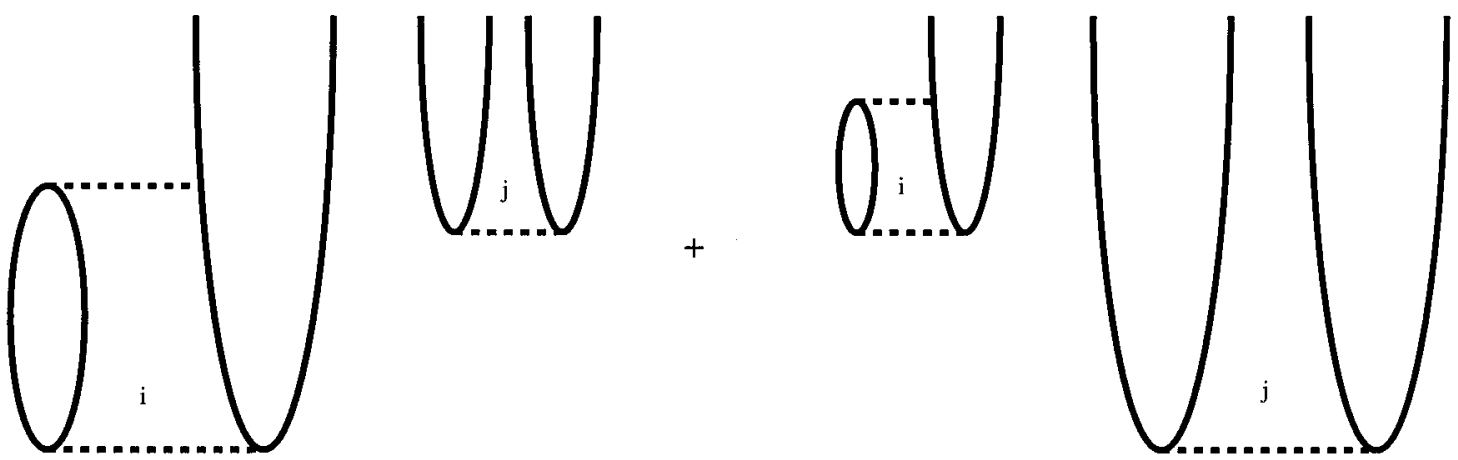

(b)

FIG. 2. (a) Connected and (b) disconnected contributions to the coefficients of the triples.

It is obvious that our scheme must have some relation with the coupled cluster method, that writes the amplitude of the quadruple excitations as product of double excitation amplitudes. A thorough comparison of both methods will be given later.

Comparison should also be done with our previous perturbative scheme. ${ }^{36}$ Due to its perturbative character, one of the two double excitations forming the quadruple was described at all-order, whereas the other one was kept at first order only. We remedy, now, this unsymmetric way of describing quadruple excitations, and thus include further higher terms. It should be noted also that the fact that no denominators appear explicitly in this new scheme is certainly in favor of its convergence properties during the iterations.

\section{B. Triple excitations}

At variance with quadruple excitations, the connected amplitudes of the triples appear before the disconnected ones. These connected triples will contribute at fourth order to the energy whereas the disconnected ones at fifth order only (see Fig. 2).

The connected triples $\left(\mathrm{T}_{\mathrm{c}}\right)$ coefficients cannot be rewritten in terms of the coefficients of the doubles and singles and thus have to be derived by perturbation upon a singly and doubly excited vector. They are single excitations of doubleexcitations.

$$
c_{\alpha}^{T_{c}}=\frac{\sum_{i}{ }^{\prime} c_{i}^{(1)}\left\langle\Phi_{\alpha}|\mathscr{H}| D_{i}^{+} \Phi_{0}\right\rangle}{\Delta_{\alpha}}
$$

where $\Sigma^{\prime}$ runs over all the double excitations for which there is no single excitation $M_{k}^{+}$such that $M_{k}^{+} D_{i}^{+} \Phi_{0}=\Phi_{\alpha}$ and $\Delta_{\alpha}$ is an energy difference to be defined by the chosen perturbative scheme (MP, EN ...). The second order relation will be generalized to an all-order relation by writing

$$
c_{\alpha}^{T_{c}}=\frac{\sum_{i}{ }^{\prime} c_{i}\left\langle\Phi_{\alpha}|\mathscr{H}| D_{i}^{+} \Phi_{0}\right\rangle}{\Delta_{\alpha}}
$$

and we have again an iterative scheme which relates the double and triple excitations.

It should be noted that the generalization is perfectly well founded. If instead of a pure single reference perturbation theory we used a multireference method, the relation (21) would be obtained directly.

Comparison with coupled cluster will show, later, that this kind of Triple contributions is obtained only by CCSDT schemes or some of its approximations.

Concerning the comparison with our previous method there is no direct improvement in the treatment of the connected triples, however the double-excitation coefficients used in Eq. (21) are of better quality due to the improvement of the $c_{\alpha}^{Q}$.

The disconnected triples $\left(\mathrm{T}_{\mathrm{dc}}\right)$ part comes from the products of disjoint single and double excitations [see Fig. 2(b)] 


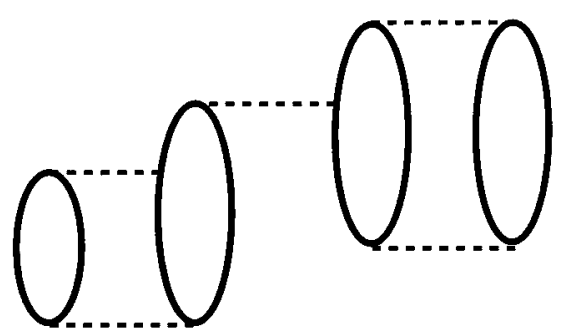

FIG. 3. Fifth order energy diagram.

$$
\Phi_{\alpha}^{T_{\mathrm{dc}}}=M_{i}^{+} D_{j}^{+} \Phi_{0}=M_{k}^{+} D_{l}^{+} \Phi_{0}=\cdots .
$$

Calling $c_{i}^{\prime}$ the coefficient of the singly excited determinant $\Phi_{i}=M_{i}^{+} \Phi_{0}$, and using again the factorization theorem one can show that this sum can be rewritten as

$$
c_{\alpha}^{T_{\mathrm{dc}}}=\sum_{(i, j)} c_{i}^{(1)^{\prime}} c_{j}^{(1)},
$$

where $(i, j)$ stands here for the nine couples of single-double excitations such that

$$
D_{j}^{+} M_{i}^{+} \Phi_{0}=\Phi_{\alpha} .
$$

This will be generalized to the obvious form

$$
c_{\alpha}^{T_{\mathrm{dc}}}=\sum_{\substack{(i, j) \\ D_{j}^{+} M_{i}^{+} \Phi_{0}=\Phi_{\alpha}}} c_{i}^{\prime} c_{j} .
$$

By including such terms in the dressing will include fifth order diagrams in the energy. One of them is shown in Fig. 3.

Such terms are automatically included if one uses natural or Brueckner orbitals instead of HF orbitals. ${ }^{45,46}$ Comparison with our previous method shows that the single excitation coefficient have changed from the first order to an all-order description. Since no denominators appear explicitly, better convergence properties can be expected during the iterations.

\section{Comparison with the coupled cluster formalism}

Let us expand $\Psi_{0}$ in Eq. (4) in a coupled cluster (CC) type way:

$$
\Psi_{0}=e^{S} \Phi_{0}
$$

If we take, for instance, the CCSD approximation, $S=T_{1}+T_{2}$ and

$$
\begin{aligned}
\Psi_{0}= & \Phi_{0}+\left(T_{1}+T_{2}+\frac{1}{2} T_{1}^{2}\right) \Phi_{0}+\left(T_{1} T_{2}+\frac{1}{6} T_{1}^{3}+\frac{1}{2} T_{1}^{2} T_{2}\right. \\
& \left.+\frac{1}{2} T_{2}^{2}+\frac{1}{24} T_{1}^{4}\right) \Phi_{0} .
\end{aligned}
$$

Written in this way, it is easy to see that each of the right hand terms in Eq. (27) has its counterpart in Eq. (4), for the case that the model space in Eq. (4) includes $\Phi_{0}$, all its Singles and all its Doubles.

Now, if we consider $\Psi_{0}$ in terms of the CI wave operator expansion and we group in the same way all excitations up to quadruples,

$$
\Psi_{0}=\Phi_{0}+\left(C_{1}+C_{2}\right) \Phi_{0}+\left(C_{3}+C_{4}\right) \Phi_{0}
$$

it follows that the procedure of taking into account the effects of the triples and quadruples on the SDCI matrix by a proper dressing $\Delta_{j}=c_{j}^{-1} \Sigma_{\alpha \notin S} c_{\alpha} h_{j \alpha}$ can be easily made equivalent to a full CCSD procedure. This is made clearer through the equivalence between the $T_{i}$ and $C_{i}$ operators

$$
\begin{aligned}
& C_{1}=T_{1}, \\
& C_{2}=T_{2}+\frac{1}{2} T_{1}^{2}, \\
& C_{3}=\frac{1}{6} T_{1}^{3}+T_{1} T_{2}, \\
& C_{4}=\frac{1}{24} T_{1}^{4}+\frac{1}{2} T_{2}^{2}+\frac{1}{2} T_{1}^{2} T_{2} .
\end{aligned}
$$

Now taking $T_{1}$ and $T_{2}$ from Eqs. (29) and (30) and substituting them into Eqs. (31) and (32), we can write

$$
\begin{aligned}
& C_{3}=C_{1} C_{2}-\frac{1}{3} C_{1}^{3}, \\
& C_{4}=\frac{1}{2} C_{2}^{2}-\frac{1}{12} C_{1}^{4},
\end{aligned}
$$

i.e., we can rewrite the CCSD, originally based solely on $T_{1}$ and $T_{2}$ amplitudes, in terms of $c_{i}$ model space wave function coefficients and $c_{\alpha}$ coefficients.

Comparison of Eqs. (33) and (34) with our $c_{\alpha}^{T_{\mathrm{dc}}}$ and $c_{\alpha}^{Q}$ will reveal the close relation between both methods. The difference is given by third and fourth powers of $C_{1}$ operator. For the triple excitations these terms will contribute at 8th order of perturbation and for the quadruple ones at the 11th order! So the difference should be very tiny, indeed! These missing terms can, of course, be included easily in our scheme but this point is not obvious. Comparison with an all-order expansion will show that these terms are only some of the numerous terms appearing at those orders. There is no a priori way to say which scheme gives the best "arbitrary weight" to these powers of $C_{1}$.

Anyway, in another work, ${ }^{47}$ we have included these $C_{1}$ powers in order to show the strict equivalence between both schemes. Nevertheless, it remains a very important difference in the practicability. Our scheme yields a diagonalization of an effective Hamiltonian (containing no energy differences) whereas the CCSD scheme is the resolution of a system of equations (containing energy differences) of which convergence is often improbable.

So far, we have only considered the disconnected terms, it remains to see how coupled cluster includes connected triple excitations. We have already said that we will not include connected quadruple excitations but their effects have been studied by Bartlett. ${ }^{48,49}$ In order to include $T_{c}$ one should use the CCSDT scheme. Such a procedure will, indeed, include the connected triples but also numerous higherorder terms yielding a method which goes as $n^{8} .{ }^{48,49}$ For most of the chemical problems such a high cost is prohibitive and approximations should be derived. The most radical one would be to decouple the $T_{c}$ amplitudes from the $T_{1}$ and $T_{2}$ ones and to calculate their contributions by a $\mathrm{MP}_{4}$ formula, using however iterative $T_{1}$ and $T_{2}$ amplitudes: this is the $\operatorname{CCSD}(\mathrm{T}) .{ }^{13-15}$ A similar method can be easily obtained with our procedure. More interesting is to keep the coupling between outer and model space as we do by having the $c_{\alpha}^{T_{c}}$ in the iterative procedure.

Rewritten in terms of coefficients but in a coupled cluster manner, we have the $c_{i}$ of the model space and 


$$
\begin{aligned}
& C_{3}=C_{1} C_{2}+C_{\alpha}^{T_{c}}+\left(-\frac{1}{3} C_{1}^{3}\right), \\
& C_{4}=\frac{1}{2} C_{2}^{2}+\left(-\frac{1}{12} C_{1}^{4}\right),
\end{aligned}
$$

the parenthesis meaning that, at convenience, the powers of $C_{1}$ should or not be included. Including all terms would give similar equations as CCSDT so the approximations are done, in fact, in the equation defining $T_{3}$ or $C_{\alpha}^{T_{c}}$. Comparing our $C_{\alpha}^{T_{c}}$ equation [Eq. (21)] with the $T_{3}$ equation as given by Bartlett $^{37}$ we can see that we include only the first term of this complicated equation. In the nomenclature of Bartlett this scheme corresponds to CCSDT- $1 a^{38}$ However, in our approach we are free to define the denominator of Eq. (21) by taking MP or EN method or to further improve this denominator by using CEPA arguments.

So, in conclusion, we will say that both methods are quite similar but that we are working with wave function coefficients and thus diagonalizing, whereas the $\mathrm{CC}$ approach uses amplitudes of excitations and must solve quite complicated systems of equations. Due to the fact that, nowadays, the diagonalization algorithms are very efficient and can be made direct, ${ }^{50,51}$ we think that our formulation opens the road to very efficient Direct methods as we propose in the present work, or to very efficient CCSDT- $n$.

\section{Proposed nomenclature}

The acronyms used in this work, which have been designed in order to take into account the great flexibility of the dressed intermediate Hamiltonian method, first indicate the Hamiltonian matrix which is iteratively dressed and diagonalized, say SDCI. Then, in rectangular braces, the terms included in the dressing: $\mathrm{T}_{\mathrm{dc}}$ and $\mathrm{Q}$ for disconnected triples and quadruples, respectively, and $\mathrm{T}$ for both connected and disconnected triples. The subindex $f$ stands for "factorization" and $p$ for "perturbation," as the way of calculating disconnected terms. Inclusion of higher order EPV terms is also indicated by adding +EPV to subindex. Angular braces indicate that the mean value of the dressed Hamiltonians has been taken instead of an iterative diagonalization procedure.

\section{E. Improvements of the $\mathrm{SDCl}[\mathrm{TQ}]_{f}$}

Our procedure can be improved in two ways. First one should consider that the factorization theorem is only correct if the energies are additive which is implicit with MöllerPlesset theory. This is a very good approximation, however there are cases where the nonadditivity can be crucial, as for example in Solid State Physics. Examples are easily found in molecular problems also. ${ }^{52}$ Second, one can think of using CEPA arguments in order to improve the description of the triple and quadruple excitations.

\section{Nonadditivity of the denominators}

If one no longer assumes the energy denominators additivity, one may move back to the perturbative evaluation of $c_{\alpha}$ as a sum of contributions as in Eq. (17), which is translated diagrammatically in Fig. 1(a). Thus,

$$
c_{\alpha}^{Q}=\frac{\sum_{(i, j)}\left(c_{i}\left\langle\Phi_{\alpha}|\mathscr{H}| \Phi_{i}\right\rangle+c_{j}\left\langle\Phi_{\alpha}\left|\mathscr{H}_{\mid}\right| \Phi_{j}\right\rangle\right)}{E_{0}^{0}-E_{\alpha}^{0}},
$$

where $(i, j)$ stands for the couples of double excitations such that

$$
D_{j}^{+} D_{i}^{+} \Phi_{0}=\Phi_{\alpha}
$$

Since

$$
\begin{aligned}
& \left\langle\Phi_{\alpha}|\mathscr{H}| \Phi_{i}\right\rangle=\left\langle\Phi_{j}|\mathscr{H}| \Phi_{0}\right\rangle, \\
& \left\langle\Phi_{\alpha}|\mathscr{H}| \Phi_{j}\right\rangle=\left\langle\Phi_{i}|\mathscr{H}| \Phi_{0}\right\rangle,
\end{aligned}
$$

and since

$$
\begin{aligned}
c_{i}^{(1)} & =\frac{\left\langle\Phi_{i}|\mathscr{H}| \Phi_{0}\right\rangle}{E_{0}^{0}-E_{i}^{0}}, \\
c_{j}^{(1)} & =\frac{\left\langle\Phi_{j}|\mathscr{H}| \Phi_{0}\right\rangle}{E_{0}^{0}-E_{j}^{0}},
\end{aligned}
$$

replacing $c_{i}$ for $c_{i}^{(1)}$ (i.e. a variational evaluation of the coefficients of the doubles for the perturbative one), Eq. (37) becomes

$$
c_{\alpha}^{Q}=\frac{\sum_{(i, j)} c_{i} c_{j}\left[\left(E_{0}^{0}-E_{i}^{0}\right)+\left(E_{0}^{0}-E_{j}^{0}\right)\right]}{E_{0}^{0}-E_{\alpha}^{0}} .
$$

In a more compact notation, calling $\Delta$ the energy differences,

$$
c_{\alpha}^{Q}=\frac{\sum_{(i, j)} c_{i} c_{j}\left(\Delta_{i}+\Delta_{j}\right)}{\Delta_{\alpha}}
$$

and similarly for the triples

$$
c_{\alpha}^{T_{\mathrm{dc}}}=\frac{\sum_{(i, j)} c_{i}^{\prime} c_{j}\left(\Delta_{i}+\Delta_{j}\right)}{\Delta_{\alpha}} .
$$

This formulation will of course use the Epstein-Nesbet definition of $\mathscr{H}_{0}$ as the diagonal of the Hamiltonian CI matrix.

\section{Inclusion of the EPV terms}

This proposal has already been formulated in the preceding paper for the perturbative dressing of the SDCI matrix by the triples and quadruples $\left(\mathrm{SDCI}[\mathrm{TQ}]_{p}\right.$, previously labeled TD1). Its justification is based on an earlier work, ${ }^{29}$ proposing a method, $(\mathrm{SC})^{2} \mathrm{CI}$, which makes size-consistent any selected CI. The coefficient $c_{\alpha}$ of any triple or quadruple $\Phi_{\alpha}$ is supposed to be obtained by considering a CI matrix where $\Phi_{\alpha}$ has been added to the SDCI space. Then the size consistency is obtained by dressing $\Phi_{\alpha}$ by the quantity $E_{\text {corr }}+\mathrm{EPV}_{\alpha}$ where $E_{\text {corr }}$ is the correlation energy and $\mathrm{EPV}_{\alpha}$ represents the effect of all double excitations which are impossible to perform on $\Phi_{\alpha}$

$$
\mathrm{EPV}_{\alpha}=-\sum_{\substack{k \\ D_{k}^{+} \Phi_{\alpha}=0}} c_{k}\left\langle\Phi_{0}|\mathscr{H}| \Phi_{k}\right\rangle .
$$

Then, the eigenequation for $\Phi_{\alpha}$ leads to the equation

$$
c_{\alpha}=\frac{\sum_{i} c_{i}\left\langle\Phi_{\alpha}|\mathscr{H}| \Phi_{i}\right\rangle}{\left\langle\Phi_{0}|\mathscr{H}| \Phi_{0}\right\rangle-\left(\left\langle\Phi_{\alpha}|\mathscr{H}| \Phi_{\alpha}\right\rangle+\mathrm{EPV}_{\alpha}\right)}
$$

The denominator including the EPV corrections may be used for the linked part of triples only, i.e., the perturbative 
residue in the here proposed $\mathrm{SDCI}[\mathrm{TQ}]_{f}$ scheme. A direct derivation of the use of this denominator may be obtained as an infinite partial summation of EPV diagrams. ${ }^{53}$

\section{F. Further improvements}

In his concluding remarks of a recent workshop devoted to the coupled cluster methods,${ }^{54}$ Kutzelnigg pointed out the necessity to imagine some flexible combinations of coupled cluster with lower cost approximations, in order to be able to treat problems with larger number of electrons and/or larger basis sets. Thinking in terms of dressing actually allows one to derive a whole series of approximations combining consistently different methods of different accuracies and costs.

\section{Combination with poorer approximations}

Suppose that approximate natural orbitals can be obtained by a low cost calculation of the density matrix (for instance, a MP2 calculation). Then, it is possible to retain the most active natural MOs (those of occupation number closer to one) in a class- 1 . The dressing of the SDCI matrix may be done as follows:

-by the $(\mathrm{SC})^{2} \mathrm{SDCI}$ procedure (taking into account the effect of the unlinked triples and quadruples)

- and by treating as a full dressing the effects of the triples and quadruples which only involve holes and particles of the class-1. Then the $n^{7}$ time-limiting step runs over a smaller number of MOs.

One may also consider a three-class partition of the NOs, putting into a class-3 the NOs whose occupation numbers are close to 2 or 0 . Then the SDCI is reduced to the determinants involving only the MOs of class- 1 and class- 2 . The other doubly excited determinants may be treated through the selfconsistently dressed independent pair excitation approximation. ${ }^{55} \mathrm{~A}$ unique vector of all coefficients obtained at different levels, is used to define EPV terms and to dress in a consistent manner the energies of the large SDCI matrix and of the small $2 \times 2$ matrices, as already proposed and practiced. $^{56}$

\section{Higher accuracy approximation}

One may of course dress a SDTCI matrix by the quadruples and pentuples as was done here for the SDCI matrix, but this will be in general impracticable, except if a preselection of MOs have been done. It seems more reasonable to add the most important triples and quadruples (or more highly excited determinants) to singles and doubles. Then it is perfectly possible to dress the selected triples and quadruples by the unlinked effects of the pentuples and hexuples, as done in the (SC) $)^{2} \mathrm{CI}$ method. The effect of the other triples and quadruples on the singles and doubles would then be added, including their linked effect, as proposed in the present work. The method would be sizeconsistent and would include the effect of the leading $T_{3}$ and $T_{4}$ operators at a very moderate cost. This procedure would allow to combine a CAS main model space with a CCSDT-1a single reference formalism and would certainly be very efficient for the study of multiple bond(s) breaking, without entering the complexity of the MRCC methods.

\section{G. Relation with other methods: Taking the mean value of dressed Hamiltonians}

In $\mathrm{CC}$ methods, the information about triples and quadruples is stocked in the wave function by means of the $e^{S}$ operator. However, effective Hamiltonians stock the information in the modified Hamiltonian matrix, and therefore we can obtain a good approximation to $\mathscr{E}_{0}$ by calculating the mean value of the $\mathbf{H}+\boldsymbol{\Delta}$ matrix, $\left\langle\tilde{\Psi}_{0}|H+\Delta| \tilde{\Psi}_{0}\right\rangle, \tilde{\Psi}_{0}$ being "good-enough" SD wave function. We can take, for instance, a (SC) $)^{2}$ SDCI wave function as it has been made by some of us previously, and will be thereafter named as $\left\langle\mathrm{SDCI}[\mathrm{TQ}]_{f}\right\rangle{ }^{35}$ This involves no more than one iteration of the CC procedure and it is accurate enough to yield values of $\mathscr{E}_{0}$ which, at equilibrium geometries, are in general better than $\operatorname{CCSD}(\mathrm{T})$ values.

\section{H. Practical implementation}

This method has been implemented in the framework of the algorithm recently proposed by Maynau and Heully ${ }^{57}$ to calculate the perturbation of the triples and quadruples over the SDCI space. This algorithm demonstrates that it is much more rapid to perform an outer loop over the triples and quadruples $\Phi_{\alpha}$ than to generate the appropriate $\Phi_{\alpha}$ 's from all couples $\left(\Phi_{i}, \Phi_{j}\right)$.Thus, for each $\Phi_{\alpha}$, we obtain all the couples $\left(\Phi_{i}, \Phi_{j}\right)$ contributing to it, we determine the coefficient $c_{\alpha}$ from the coefficients $c_{i}$ and $c_{j}$, and then the contribution of $\Phi_{\alpha}$ to the dressing $\Delta_{i}$ and $\Delta_{j}$ of all the couples $\left(\Phi_{i}, \Phi_{j}\right)$.

The algorithm proceeds as follows:

(1) Start: A conventional SDCI to get the initial $c_{i}$ 's.

(2) Loop over Triples and Quadruples: Calculation of $c_{\alpha}$; calculation of $\boldsymbol{\Delta}$.

(3) Building and diagonalization of $\mathbf{H}+\boldsymbol{\Delta}$.

(4) Comparison of the $\mathscr{E}_{0}$ with the previous value. If convergence is not achieved, one comes back to 2 with the new $c_{i}$ 's.

If only the dressing of the doubles by the quadruples is taken into account the algorithm results in an iterative CCD diagonalization scheme. If the unlinked contributions to the triples are included too, the dressing of the singles by the triples is taken into account, and then the algorithm results in an iterative CCSD diagonalization scheme. The inclusion of the linked contributions to the triples (and the EPV's) by means of the Epstein-Nesbet partition results in a method similar to CCSDT-1a, which we name as $\mathrm{SDCI}[\mathrm{TQ}]_{f}$ (or $\mathrm{SDCI}[\mathrm{TQ}]_{f+\mathrm{EPV}}$ ) always in a form of iterative diagonalizations. EPV's have been implemented through the introduction of one-, two-, and three-dimensional arrays, ${ }^{29}$ which is based on a previous work on the infinite summation of EPV diagrams. ${ }^{53}$

The algorithm in its present form has the following advantages:

(1) It converges even where conventional CCD, CCSD, and $\operatorname{CCSD}(\mathrm{T})$ does not, for instance, at large interatomic distances in a single or two single bond dissociation process. 
TABLE I. Differences (millihartrees) with FCI energies in BH (DZ) and $\mathrm{H}_{2} \mathrm{O}$ (DZ and DZP).

\begin{tabular}{|c|c|c|c|c|c|c|c|c|c|}
\hline \multirow[b]{2}{*}{ Method } & \multicolumn{3}{|c|}{$\mathrm{BH}(\mathrm{DZP})$} & \multicolumn{3}{|c|}{$\mathrm{H}_{2} \mathrm{O}(\mathrm{DZ})$} & \multicolumn{3}{|c|}{$\mathrm{H}_{2} \mathrm{O}$ (DZP) } \\
\hline & $R_{e}$ & $1,5 R_{e}$ & $2 R_{e}$ & $R_{e}$ & $1,5 R_{e}$ & $2 R_{e}$ & $R_{e}$ & $1,5 R_{e}$ & $2 R_{e}$ \\
\hline $\mathrm{CCSD}^{\mathrm{a}}$ & 1,78 & 2,64 & 5,04 & 1,79 & 5,59 & 9,33 & 4,12 & 10,16 & 21,4 \\
\hline $\mathrm{SDCI}\left[\mathrm{T}_{\mathrm{dc}} \mathrm{Q}\right]_{f}$ & 1,78 & 2,64 & 5,03 & 1,79 & 5,59 & 9,55 & 4,12 & 10,16 & 21,5 \\
\hline $\operatorname{CCSD}(\mathrm{T})$ & $0,41^{\mathrm{b}}$ & $0,55^{\mathrm{b}}$ & $0,41^{\mathrm{b}}$ & 0,57 & 1,47 & $-7,70$ & $0,72^{\mathrm{b}}$ & $2,00^{\mathrm{b}}$ & $-4,64^{\mathrm{b}}$ \\
\hline CCSDT- $1 \mathrm{a}^{\mathrm{a}}$ & 0,45 & 0,61 & 0,72 & 0,45 & 1,46 & $-5,58$ & 0,60 & 1,99 & $-2,65$ \\
\hline $\mathrm{SDCI}[\mathrm{TQ}]_{f}$ & $-0,15$ & $-0,34$ & $-1,22$ & 0,06 & $-0,38$ & $-15,11$ & $-0,29$ & $-1,04$ & $-14,47$ \\
\hline $\mathrm{SDCI}[\mathrm{TQ}]_{f+\mathrm{EPV}}$ & $-0,07$ & $-0,18$ & $-0,68$ & 0,11 & 0,11 & $-10,29$ & $-0,14$ & $-0,25$ & $-9,70$ \\
\hline $\mathrm{SDCI}[\mathrm{TQ}]_{f(\mathrm{MP})}$ & 0,46 & 0,63 & 1,02 & $\ldots$ & $\ldots$ & $\ldots$ & 0,60 & 2,01 & $-2,51$ \\
\hline $\operatorname{CCSDT}^{\mathrm{a}}$ & 0,06 & 0,02 & 0,02 & 0,44 & 1,52 & $-1,87$ & 0,53 & 1,83 & $-2,17$ \\
\hline $\operatorname{CC5SD}\left(\mathrm{TQ}^{*}\right)^{\mathrm{c}}$ & 0,05 & 0,01 & $-0,59$ & $\ldots$ & $\ldots$ & $\ldots$ & 0,19 & 0,13 & $-1,96$ \\
\hline $\operatorname{CCSDTQ}^{\mathrm{d}}$ & 0,00 & 0,00 & $+0,00$ & $\ldots$ & $\ldots$ & .. & 0,02 & 0,14 & $-0,02$ \\
\hline
\end{tabular}

(2) At equilibrium distances the number of iterations to converge within $1 \mu \mathrm{H}$ is typically of 4 to 6 .

(3) Its possibility to take benefit of the parallel machines. Slave processors can calculate independently the contributions of different triples or quadruples to Dressing matrix $\Delta .{ }^{58}$ Moreover, a parallel algorithm to diagonalize large matrices is in progress. Thus, the possibility to calculate very large systems, or to use very large basis sets is open.

The main disadvantage of this algorithm in its present implementation is that it grows as $n^{8}$, although it could take benefit of the same reduction to $n^{6}$ or $n^{7}$ that can be achieved on CCSD or CCSDT-1a methods. ${ }^{19}$ Test calculations on $\mathrm{H}_{2} \mathrm{O}$ with a DZ basis set shows that the present implementation of the $\mathrm{SDCI}[\mathrm{TQ}]_{f}$ is three times faster than full CCSDT, but three times slower than CCSDT-1a one, the CC calculations being performed with the Scuseria's series of programs. ${ }^{59}$

\section{NUMERICAL RESULTS AND DISCUSSION}

We have applied our methodology to a few examples. Two of them are classic comparisons with benchmark calculations, the other two refer to 4-electron systems previously presented: ${ }^{36}$ the square to rectangular deformation of the $\mathrm{H}_{4}$ system, and the breaking of a single bond in presence of an electron pair, in the $\mathrm{T}$-shaped $\mathrm{Li}_{4}$ system. These systems represent situations where a degeneracy appears between $\Phi_{0}$ and one of the doubles. We present also the results on another 4-electron system which dissociates into two closed shell atoms, the $\mathrm{Be}_{2}$ molecule.

\section{A. Benchmark calculations: $\mathrm{BH}$ and $\mathrm{H}_{2} \mathrm{O}$ molecules}

As a first application of the method we have considered the BH molecule. The reference calculations are those DZP FCI ones at $R_{e}$ from Ref. 60, and at 1,5 $R_{e}$ and $2 R_{e}$ from Ref. 16. The second application concerns to $\mathrm{H}_{2} \mathrm{O}$ molecule with a double- $\zeta(\mathrm{DZ})$ and double- $\zeta$ plus polarization basis set (DZP). Comparisons are made with the benchmark calculations at DZ FCI level at $R_{e}$ by Saxe et al. ${ }^{61}$ and at $1,5 R_{e}$ and $2 R_{e}$ by Harrison et al. ${ }^{60}$ and at DZP level the frozen core FCI calculations at the same three distances by Bauschlicher et al. ${ }^{62}$ The differences in $\mathrm{mH}$ relative to FCI values are shown in Table I. The values for conventional CC methods are taken from Refs. 14, 16, 48, and 49. The perturbative inclusion of the triples in an iterative manner, by means of the Epstein-Nesbet partition (results marked as $\mathrm{SDCI}[\mathrm{TQ}]_{f}$ ) shows the trends expected from this approach. Since higher order effects are included, the correlation energies lies under the FCI value in almost all the cases considered. However, the values at short distances are closer to FCI than the corresponding Möller-Plesset CCSDT-1a ones. Inclusion of the EPV higher order effects makes the results (named as SDCI $[\mathrm{TQ}]_{f+\mathrm{EPV}}$ ) closer to FCI. In fact, at short distances, the SDCI $[\mathrm{TQ}]_{f+\mathrm{EPV}}$ results are comparable to full CCSDT ones. However, at $2 R_{e}$, both SDCI[TQ $]_{f}$ and $\mathrm{SDCI}[\mathrm{TQ}]_{f+\mathrm{EPV}}$ methods give values far from $\mathrm{FCI}$ results. Table I shows also the DZP results for $\mathrm{BH}$ and $\mathrm{H}_{2} \mathrm{O}$ molecules when the linked contributions to triples are taken into account within the Möller-Plesset partition, marked as $\mathrm{SDCI}[\mathrm{TQ}]_{f(\mathrm{MP})}$. It is to note that the results of $\mathrm{SDCI}[\mathrm{TQ}]_{f(\mathrm{MP})}$ calculations are almost identical to CCSDT-1a ones near the equilibrium distances. At long distances the differences can be related to the different treatment of the $T_{1}$ terms. When one takes into account only the effects of the nonlinked contributions to triples and quadruples (named as $\mathrm{SDCI}\left[\mathrm{T}_{\mathrm{dc}} \mathrm{Q}\right]_{f}$ ), the values obtained at $R_{e}$ and 1,5 $R_{e}$ are the same as those obtained with CCSD, but small differences appear at $2 R_{e}$ in the water molecule. These differences could be related with the absence of terms including powers of $T_{1}$, which are important when polar single bonds are simultaneously broken.

\section{B. The rectangular to square $\mathrm{H}_{4}$ problem}

This model problem has been a critical test for many methods, especially for CC methods. ${ }^{63-65}$ Along the rectangular to square transformation for $\mathrm{H}_{4}$ system a degeneration appears between the $\Phi_{0}$ determinant and another doubly excited determinant. Geometry has been explicited elsewhere. ${ }^{36}$ The calculations have been performed with a double- $\zeta$ basis set. Table II shows the energy differences relatives to FCI 
TABLE II. Rectangular $\mathrm{H}_{4}$ system in a double zeta basis set as a function of $R$ (bohr) for $r=1.40158$ bohr. FCI energies in hartrees and differences to FCI in millihartrees.

\begin{tabular}{|c|c|c|c|c|c|c|}
\hline$R$ & $E(\mathrm{FCI})$ & $\mathrm{SDCI}[\mathrm{TQ}]_{f}$ & $\mathrm{SDCI}[\mathrm{TQ}]_{f+\mathrm{EPV}}$ & $\left\langle\mathrm{SDCI}[\mathrm{T}]_{p}[\mathrm{Q}]_{f}\right\rangle$ & $\operatorname{CCSD}(\mathrm{T})$ & QSDCI(T) \\
\hline 1,20 & $-1,793989$ & 0,00 & 0,01 & 0,05 & 0,16 & 0,17 \\
\hline 1,24 & $-1,802647$ & $-0,02$ & 0,00 & 0,07 & 0,23 & 0,24 \\
\hline 1,28 & $-1,810103$ & $-0,06$ & $-0,02$ & 0,11 & 0,34 & 0,35 \\
\hline 1,32 & $-1,817367$ & $-0,18$ & $-0,11$ & 0,16 & 0,48 & 0,51 \\
\hline 1,36 & $-1,826043$ & $-0,54$ & $-0,39$ & 0,17 & 0,45 & 0,54 \\
\hline 1,40 & $-1,838209$ & $-1,42$ & $-1,11$ & $-0,13$ & $-1,04$ & $-0,77$ \\
\hline 1,40158 & $-1,838784$ & $-1,47$ & $-1,15$ & $-0,16$ & $-1,17$ & $-0,89$ \\
\hline 1,42 & $-1,846002$ & $-1,01$ & $-0,77$ & 0,03 & $-0,13$ & 0,05 \\
\hline 1,44 & $-1,854827$ & $-0,65$ & $-0,49$ & 0,11 & 0,32 & 0,43 \\
\hline 1,46 & $-1,864440$ & $-0,42$ & $-0,31$ & 0,13 & 0,43 & 0,50 \\
\hline 1,48 & $-1,874579$ & $-0,28$ & $-0,19$ & 0,12 & 0,42 & 0,47 \\
\hline 1,50 & $-1,885016$ & $-0,19$ & $-0,12$ & 0,11 & 0,38 & 0,41 \\
\hline 1,52 & $-1,895580$ & $-0,13$ & $-0,08$ & 0,09 & 0,33 & 0,35 \\
\hline 1,54 & $-1,906151$ & $-0,09$ & $-0,05$ & 0,08 & 0,28 & 0,29 \\
\hline 1,56 & $-1,916645$ & $-0,06$ & $-0,03$ & 0,07 & 0,24 & 0,25 \\
\hline 1,58 & $-1,927007$ & $-0,04$ & $-0,02$ & 0,06 & 0,21 & 0,21 \\
\hline 1,60 & $-1,937201$ & $-0,03$ & $-0,01$ & 0,06 & 0,18 & 0,18 \\
\hline 1,80 & $-2,027364$ & 0,00 & 0,01 & 0,03 & 0,07 & 0,07 \\
\hline 2,00 & $-2,096821$ & 0,00 & 0,01 & 0,03 & 0,04 & 0,04 \\
\hline 2,20 & $-2,149662$ & 0,00 & 0,01 & 0,02 & 0,03 & 0,03 \\
\hline 2,40 & $-2,189660$ & 0,00 & 0,01 & 0,02 & 0,02 & 0,02 \\
\hline 2,60 & $-2,219768$ & 0,00 & 0,00 & 0,02 & 0,02 & 0,02 \\
\hline 2,80 & $-2,242283$ & 0,00 & 0,00 & 0,02 & 0,01 & 0,02 \\
\hline 20,00 & $-2,303002$ & 0,01 & 0,01 & 0,00 & 0,00 & 0,00 \\
\hline
\end{tabular}

values in millihartrees calculated at $\mathrm{SDCI}[\mathrm{TQ}]_{f}$, $\mathrm{SDCI}[\mathrm{TQ}]_{f+\mathrm{EPV}}$, and $\left\langle\mathrm{SDCI}[\mathrm{T}]_{p}[\mathrm{Q}]_{f}\right\rangle$ levels of theory and compared with the $\operatorname{CCSD}(\mathrm{T})$ and $\mathrm{QSDCI}(\mathrm{T})$ values for different intermolecular distances $R$. All these results are also displayed in Fig. 4. The behaviour of the SDCI[TQ $]_{f}$ and $\mathrm{SDCI}[\mathrm{TQ}]_{f+\mathrm{EPV}}$ methods is very similar, with the $\mathrm{SDCI}[\mathrm{TQ}]_{f+\mathrm{EPV}}$ values slightly closer to FCI results. At the degeneracy point, all the iterative methods give nearly the

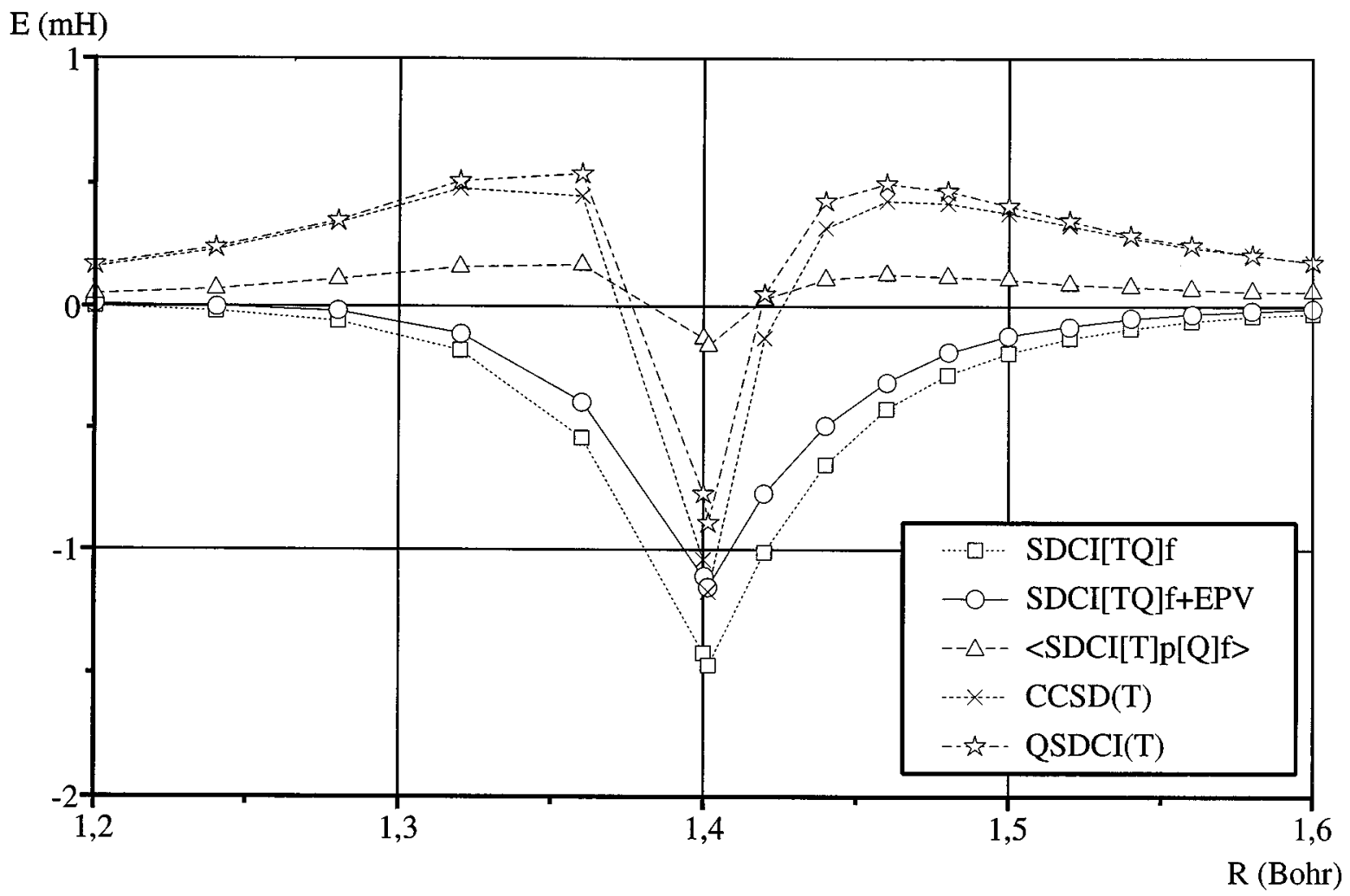

FIG. 4. Energy differences in $\mathrm{mH}$ relative to FCI in the $\mathrm{H}_{4}$ problem in a double- $\zeta$ basis set, as a function of $R$ for $r=1.40158$ a.u. 
TABLE III. T-shaped $\mathrm{Li}_{4}$ system in an ANO $[2 s, 1 p]$ basis set as a function of the axial bond length, $R$ (in bohr). FCI energies in hartrees and differences to FCI in millihartrees.

\begin{tabular}{ccccccc}
\hline \hline$R$ & $E(\mathrm{FCI})$ & $\left.\mathrm{SDCI}_{\mathrm{TTQ}}\right]_{f}$ & $\mathrm{SDCI}[\mathrm{TQ}]_{f+\mathrm{EPV}}$ & $\mathrm{CCSD}(\mathrm{T})$ & $\mathrm{QSDCI}(\mathrm{T})$ & $\left\langle\mathrm{SDCI}[\mathrm{T}]_{p}[\mathrm{Q}]_{f}\right\rangle$ \\
\hline 4,00 & $-29,772243$ & $-0,31$ & $-0,11$ & $\ldots$ & $\ldots$ & 0,16 \\
4,40 & $-29,783253$ & $-0,30$ & $-0,11$ & $\ldots$ & $\ldots$ & 0,13 \\
4,80 & $-29,789148$ & $-0,31$ & $-0,10$ & 0,49 & $\ldots$ & 0,10 \\
5,00 & $-29,790673$ & $-0,31$ & $-0,11$ & 0,51 & 0,49 & 0,09 \\
5,20 & $-29,791469$ & $-0,32$ & $-0,10$ & 0,53 & 0,51 & 0,09 \\
5,40 & $-29,791674$ & $-0,33$ & $-0,10$ & 0,55 & 0,53 & 0,08 \\
5,60 & $-29,791404$ & $-0,34$ & $-0,10$ & 0,57 & 0,55 & 0,07 \\
5,80 & $-29,790761$ & $-0,35$ & $-0,10$ & 0,60 & 0,57 & 0,07 \\
6,00 & $-29,789830$ & $-0,36$ & $-0,10$ & 0,63 & 0,60 & 0,07 \\
6,20 & $-29,788681$ & $-0,38$ & $-0,10$ & 0,66 & 0,63 & 0,07 \\
6,40 & $-29,787375$ & $-0,40$ & $-0,10$ & 0,69 & 0,67 & 0,08 \\
6,60 & $-29,785964$ & $-0,42$ & $-0,10$ & 0,73 & 0,71 & 0,08 \\
6,80 & $-29,784489$ & $-0,44$ & $-0,10$ & 0,77 & 0,75 & 0,08 \\
7,00 & $-29,782986$ & $-0,48$ & $-0,10$ & 0,81 & 0,79 & 0,09 \\
7,20 & $-29,781484$ & $-0,51$ & $-0,10$ & 0,86 & 0,84 & 0,09 \\
7,40 & $-29,780007$ & $-0,56$ & $-0,11$ & 0,91 & 0,89 & 0,09 \\
7,60 & $-29,778575$ & $-0,61$ & $-0,11$ & 0,96 & 0,94 & 0,09 \\
7,80 & $-29,777203$ & $-0,68$ & $-0,12$ & 1,01 & 0,99 & 0,09 \\
8,00 & $-29,775901$ & $-0,75$ & $-0,13$ & 2,52 & 1,04 & 0,08 \\
9,00 & $-29,770660$ & $-1,32$ & $-0,25$ & 3,31 & 1,24 & $-0,05$ \\
10,00 & $-29,767460$ & $-2,17$ & $-0,46$ & 1,19 & 1,22 & $-0,35$ \\
20,00 & $-29,763831$ & $-7,94$ & $-1,67$ & $\mathrm{a}$ & $\mathrm{a}$ & $-2,97$ \\
40,00 & $-29,763798$ & $-8,92$ & $-1,73$ & $\mathrm{a}$ & $\mathrm{a}$ & $-3,32$ \\
100,00 & $-29,763798$ & $-9,68$ & $-1,76$ & $\mathrm{a}$ & $\mathrm{a}$ & $-3,54$ \\
\hline \hline
\end{tabular}

${ }^{a}$ Method does not converge.

same error $(0,9$ to $1,5 \mathrm{mH})$, but for all other distances, out of the $R=r$ point, the $\mathrm{SDCI}[\mathrm{TQ}]_{f}$ and $\mathrm{SDCI}[\mathrm{TQ}]_{f+\mathrm{EPV}}$ methods remain closer to $\mathrm{FCI}$ values than $\operatorname{CCSD}(\mathrm{T})$ and $\operatorname{QSDCI}(\mathrm{T})$ ones. Moreover, their behaviour is also more regular, since the deviation from FCI has the same sign for all values of $R$. The shape of the error curve for the mean value $\mathrm{SDCI}[\mathrm{TQ}]_{f}$ method $\left(\left\langle\mathrm{SDCI}[\mathrm{T}]_{p}[\mathrm{Q}]_{f}\right\rangle\right),{ }^{35}$ is similar to that of $\operatorname{CCSD}(\mathrm{T})$ or QSDCI(T) ones, but its mean error is smaller. We point out that, for this system the errors reported in the present work, in the degeneracy region, are larger than those obtained from the SDCI $[\mathrm{TQ}]_{p}$ methods, ${ }^{36}$ where the coefficients of the triples and quadruples are estimated perturbatively. At the degeneracy point $(r=R)$ these errors were 0,5 $\mathrm{mH}$ for the method SDCI[TQ $]_{p}$ and $0,09 \mathrm{mH}$ for the method $\mathrm{SDCI}[\mathrm{TQ}]_{p+\mathrm{EPV}}$. The small errors in $\mathrm{SDCI}[\mathrm{TQ}]_{p+\mathrm{EPV}}$ method could be explained because in it, higher-order EPV corrections are included for all contributions to triples and quadruples.

\section{The T-shaped $\mathrm{Li}_{4}$ cluster}

The geometric parameters describing the breaking of a single bond in the T-shaped $\mathrm{Li}_{4}$ cluster four electron system have been described in the previous work. ${ }^{36}$ Calculations have been carried out with a DZP $[2 s, 1 p]$ ANO's basis set. ${ }^{66}$ The values for the four electron FCI total energy and the differences from the FCI values are shown in Table III, and in Fig. 5 the potential energy curve for the dissociation process is displayed. The errors of the $\mathrm{SDCI}[\mathrm{TQ}]_{f}$ method with relation to FCI are of the same order or somewhat lesser than those of $\operatorname{CCSD}(\mathrm{T})$ or QSDCI(T) methods, although of opposite sign, due to the Epstein-Nesbet choice of the zeroth order Hamiltonian. When higher order EPV corrections are included in the linked contributions to triples, the errors fall to $-0,1 \mathrm{mH}$ in almost all the range of values of $R$. Note here the convergence of our algorithm, based on iteratively diagonalizing a dressed SDCI matrix, by comparison with CC or QCI methods which fail to converge for large values of $R$. $\mathrm{SDCI}[\mathrm{TQ}]_{f}$ curve (Fig. 5) shows the typical behaviour of the $\mathrm{CC}$ dissociation curves, where dissociation limit lies far below the FCI dissociation limit. The EPV terms avoid the most of this deficiency, since they increase the resistance to the degeneracies occurring at long bond distances.

\section{The $\mathrm{Be}_{2}$ molecule}

$\mathrm{Be}_{2}$ molecule is a very challenging system for theoretical methods. ${ }^{37,67}$ Despite the closed shell character of the $\mathrm{Be}$ atom, a true chemical bond is formed between two Be atoms at short distances. ${ }^{68}$ Most of the theoretical methods fail to represent this bond, and a good basis set is necessary. ${ }^{67}$ Figure 6 shows the potential energy curves calculated with four electrons and a $[3 s, 2 p, 1 d]$ ANO basis set, ${ }^{66}$ and FCI, $\operatorname{CCSD}(\mathrm{T})$ and the SDCI $[\mathrm{TQ}]_{f}$ methods. The FCI energies and the differences to FCI energies for these methods are shown in Table IV. The more striking feature is the inability of the $\operatorname{CCSD}(\mathrm{T})$ method to merely reproduce a bond between the two atoms with this basis set. The importance of the linked triples is very large for this system, and they are included by means of a MP single calculation in $\operatorname{CCSD}(\mathrm{T})$ method. If the linked triples are taken into account by an iterative EN procedure, as in the $\mathrm{SDCI}[\mathrm{TQ}]_{f}$ method, the main well at short lengths appears, but the bond is overestimated, and the minimum well is too deep. When higher- 


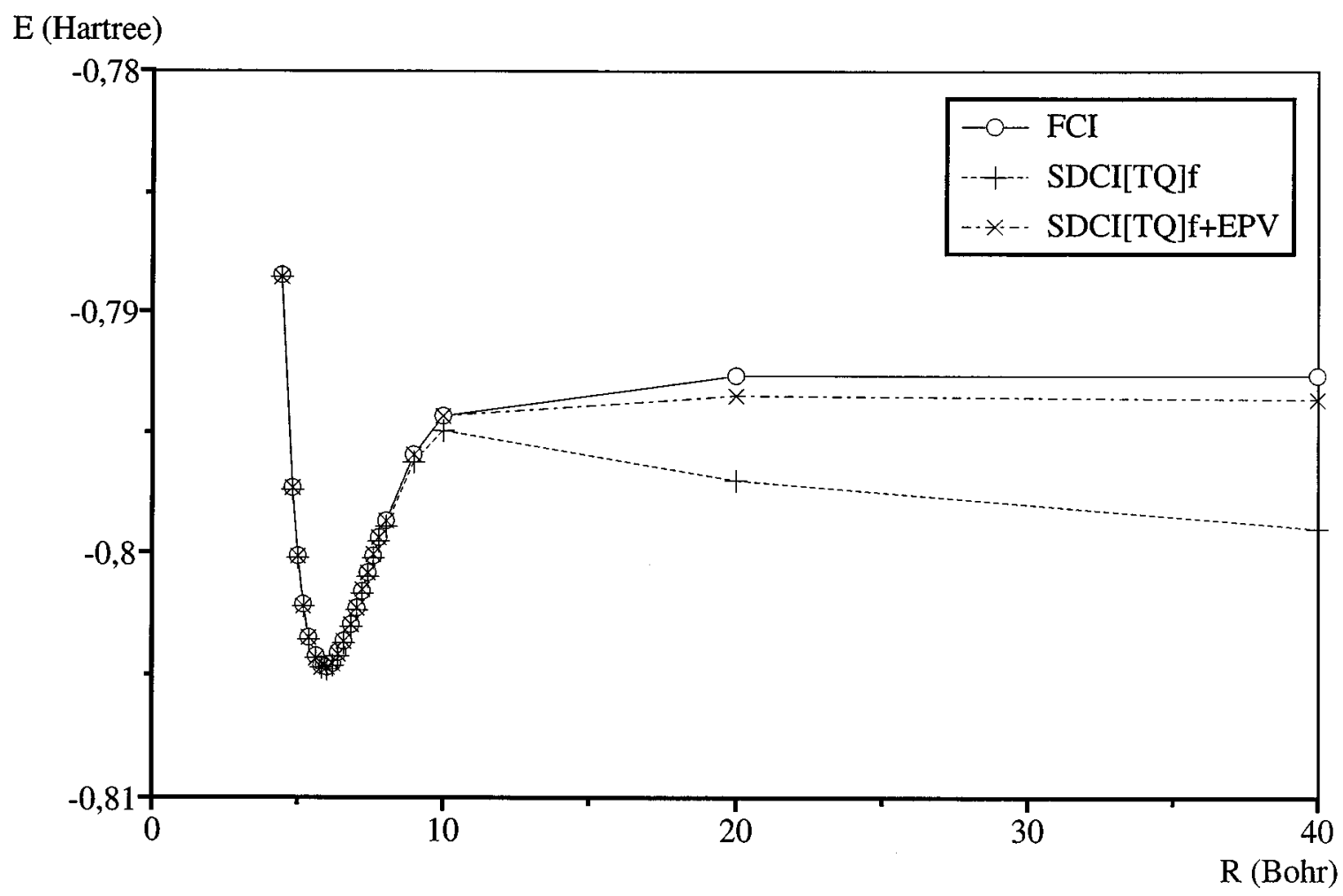

FIG. 5. Evolution of the energy in the T-shaped $\mathrm{Li}_{4}$ problem in a $[2 s, 1 \mathrm{p}]$ ANO basis set, as a function of the length $R$ of the axial bond.

order EPV's are included, as in the SDCI[TQ $]_{f+E P V}$ method, the resulting curve is almost identical to the FCI one, and the errors, near $R_{e}$, are equal to $0,02 \mathrm{mH}$ or smaller, 2 orders of magnitude less than $\operatorname{CCSD}(\mathrm{T})$ ones. At long distances, the three methods converge towards the FCI dissociation limit. It is to note the $\left\langle\mathrm{SDCI}[\mathrm{T}]_{p}[\mathrm{Q}]_{f}\right\rangle$ results, which are fairly parallel to the FCI curve, although the errors at the dissociation limit are somehow larger that in the minimum region.

\section{E (Hartree)}

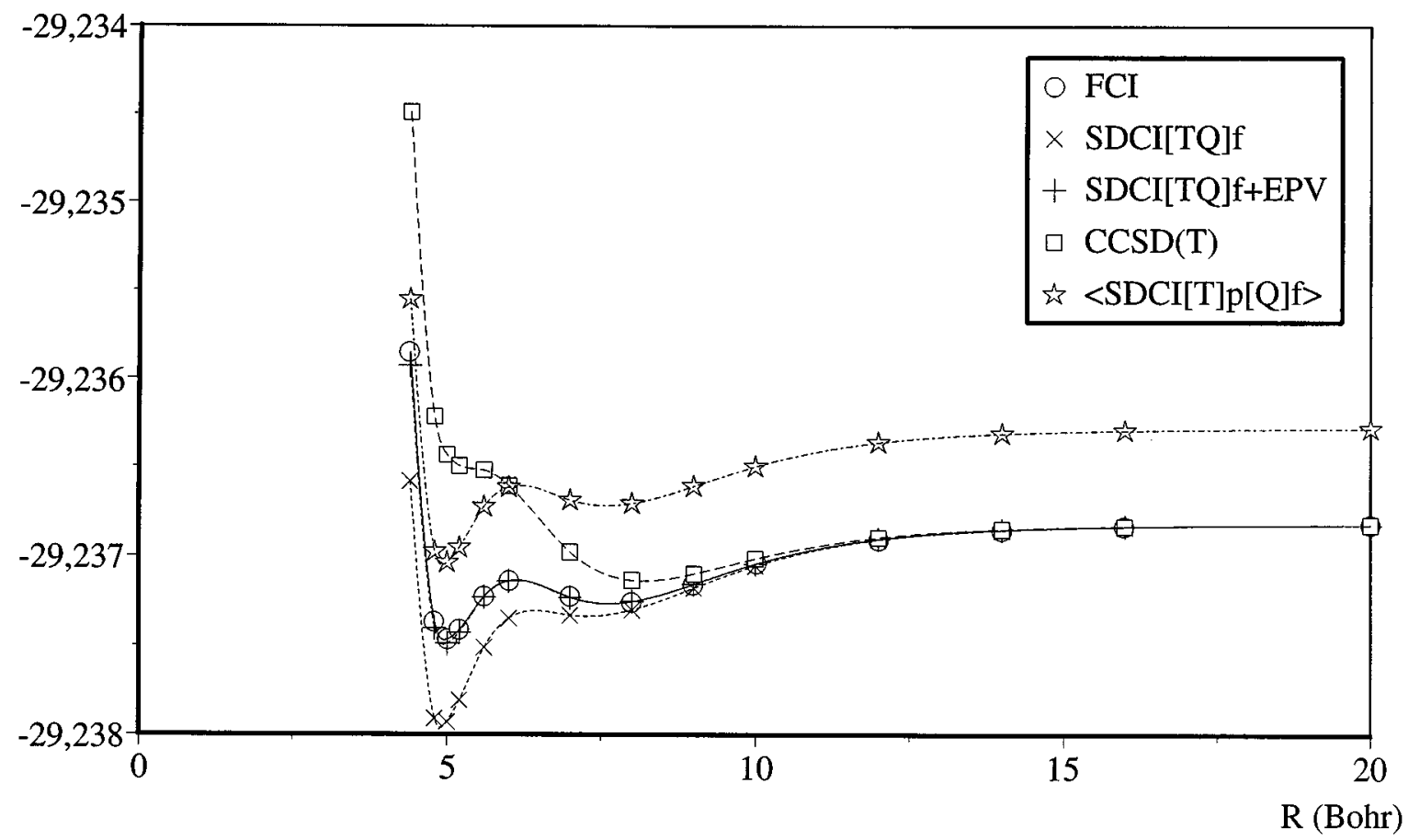

FIG. 6. Evolution of the energy in the $\mathrm{Be}_{2}$ molecule in a $[3 s, 2 p, 1 d]$ ANO basis set, as a function of the bond length, $R$. 
TABLE IV. $\mathrm{Be}_{2}$ molecule in an ANO $[3 s, 2 p, 1 d]$ basis set as a function of the internuclear distance, $R$ (in bohr). FCI energies in hartrees and differences to FCI in millihartrees.

\begin{tabular}{lccccc}
\hline \hline$R$ & $E(\mathrm{FCI})$ & $\mathrm{SDCI}^{\prime}[\mathrm{TQ}]_{f}$ & $\left.\mathrm{SDCI}_{\mathrm{cTQ}}\right]_{f+\mathrm{EPV}}$ & $\mathrm{CCSD}(\mathrm{T})$ & $\left\langle\mathrm{SDCI}[\mathrm{T}]_{p}[\mathrm{Q}]_{f}\right\rangle$ \\
\hline 4 & $-29,229824$ & $-0,92$ & $-0,12$ & 1,51 & 0,23 \\
4,4 & $-29,235853$ & $-0,73$ & $-0,08$ & 1,36 & 0,30 \\
4,8 & $-29,237368$ & $-0,54$ & $-0,04$ & 1,15 & 0,39 \\
5 & $-29,237467$ & $-0,46$ & $-0,02$ & 1,03 & 0,43 \\
5,2 & $-29,237414$ & $-0,39$ & $-0,01$ & 0,92 & 0,46 \\
5,6 & $-29,237227$ & $-0,28$ & 0,00 & 0,71 & 0,50 \\
6 & $-29,237140$ & $-0,21$ & 0,00 & 0,53 & 0,52 \\
7 & $-29,237231$ & $-0,10$ & 0,00 & 0,25 & 0,54 \\
8 & $-29,237255$ & $-0,05$ & 0,00 & 0,12 & 0,55 \\
9 & $-29,237158$ & $-0,02$ & 0,00 & 0,06 & 0,55 \\
10 & $-29,237044$ & $-0,01$ & 0,00 & 0,03 & 0,55 \\
12 & $-29,236905$ & 0,00 & 0,00 & 0,01 & 0,54 \\
14 & $-29,236853$ & 0,00 & 0,00 & 0,00 & 0,54 \\
16 & $-29,236834$ & 0,00 & 0,00 & 0,00 & 0,54 \\
20 & $-29,236824$ & 0,00 & 0,00 & 0,00 & 0,54 \\
\hline \hline
\end{tabular}

\section{CONCLUDING REMARKS}

Within the intermediate Hamiltonian framework, we have formulated a total dressing of a SDCI Hamiltonian matrix for a closed-shell single reference. The present formulation, more general than $\mathrm{CI}$ or CC methods, includes these as particular cases, and allows an easy extension of the $\mathrm{CC}$ series. Also, the flexibility in the definition of the dressing matrix allows for the definition of mixed approximated methods or selected CC-like methods. Moreover, the possibility of dealing with MRCI matrices open the door for MR-CC-like formalisms. Since the method proceeds by diagonalization of the SDCI matrix, the effect of the quadruples and the triples on all the single and doubly excited determinants includes an infinite partial summation of higher order diagrams. An estimation of the coefficients of the nonlinked triples and quadruples in a coupled cluster fashion results in an iterative method which includes all the fourth order, and which differs from the CCSD method in the lack of the terms including third and fourth powers of the $T_{1}$ (or $C_{1}$ ) operator. If the linked contributions to triples are included perturbatively, the resulting method is similar to the previous CCSDT-1a, but differing in the Epstein-Nesbet choice of the unperturbed Hamiltonian. This difference is evidenced in the slight overestimation of the correlation energy, which is a characteristic of this choice. Inclusion of higher order EPV's correction is trivial in EN framework, and the resulting method compares well with CCSDT except for long distances when two single bonds are broken simultaneously, as in the $\mathrm{H}_{2} \mathrm{O}$ dissociation process. The numerical stability of the algorithm is also remarkable, based in an hermitian row and column dressing and the diagonalization of a SDCI dressed matrix, which allows it to converge even when traditional CC algorithms cannot. The present implementation of this algorithm involves a loop over all the triples and quadruples, and therefore the time of calculation grows as $n^{8}$. However, it has been completely parallelized, and it could take benefit of the same intermediate summations which make CCSD a $n^{6}$ algorithm. Numerical applications on some model systems show that the combination of
EN zeroth order Hamiltonian and EPV higher order corrections give very good estimations of the FCI correlation energy.

\section{ACKNOWLEDGMENTS}

The research reported in this paper has been supported by the DGICYT Project No. PB91-0634, the SEUI Grant No. OP90-0042, and a grant from Conselleria de Cultura, Educació i Ciència de la Generalitat Valenciana. Authors are indebted to Professor G. Scuseria and B.O. Roos who kindly has sent to us their CC and MOLCAS-2 series of programs, respectively. Computational support from the C.I.U.V. of Universitat de València is also acknowledged.

\footnotetext{
${ }^{1}$ K. A. Brueckner, Phys. Rev. 97, 1353 (1953).

${ }^{2}$ K. A. Brueckner, Phys. Rev. 100, 36 (1955).

${ }^{3}$ H. Primas, in Modern Quantum Chemistry, edited by O. Sinanoglu (Academic, New York, 1961), Vol. 2, p. 45.

${ }^{4}$ W. Kutzelnigg, in Modern Theoretical Chemistry, edited by H. F. Schaefer (Plenum, New York, 1977), Vol. 2.

${ }^{5}$ A. Szabo and N. S. Ostlund, Modern Quantum Chemistry. Introduction to Advanced Electronic Structure Theory, (McGraw-Hill, New York, 1989).

${ }^{6}$ F. Coestler, Nucl. Phys. 1, 421 (1958).

${ }^{7}$ F. Coestler and H. Kummel, Nucl. Phys. 17, 477 (1960).

${ }^{8}$ J. Cizek, Adv. Chem. Phys. 14, 35 (1969).

${ }^{9}$ J. Cizek and J. Paldus, Phys. Scr. 21, 251 (1980).

${ }^{10}$ R. J. Bartlett, Ann. Rev. Phys. Chem. 32, 359 (1981).

${ }^{11}$ R. J. Bartlett, J. Phys. Chem. 93, 1697 (1989).

${ }^{12} \mathrm{~J}$. Paldus, in Methods in Computational Molecular Physics, edited by S. Wilson and G. H. F. Dierksen (Plenum, New York, 1992), p. 99.

${ }^{13}$ K. Raghavachari, G. W. Trucks, J. A. Pople, and M. Head-Gordon, Chem. Phys. Lett. 157, 479 (1989).

${ }^{14}$ R. J. Bartlett, J. D. Watts, S. A. Kucharski, and J. Noga, Chem. Phys. Lett. 165, 513 (1990).

${ }^{15}$ R. J. Bartlett, J. D. Watts, S. A. Kucharski, and J. Noga, Chem. Phys. Lett. 167, 609 (1990).

${ }^{16}$ J. Noga and R. J. Bartlett, J. Chem. Phys. 86, 7041 (1987).

${ }^{17}$ J. Noga and R. J. Bartlett, J. Chem. Phys. 89, 3401 (1988).

${ }^{18}$ G. E. Scuseria and H. F. Schaefer III, Chem. Phys. Lett. 152, 382 (1988).

${ }^{19}$ G. E. Scuseria, C. L. Janssen, and H. F. Schaefer III, J. Chem. Phys. 89, 7382 (1988).

${ }^{20}$ P. Piecuch and L. Adamowich, J. Chem. Phys. 100, 5857 (1994).

${ }^{21}$ H. P. Kelly and M. A. Sessler, Phys. Rev. 132, 2091 (1963).

${ }^{22}$ H. P. Kelly and M. A. Sessler, Phys. Rev. A 134, 1450 (1964).

${ }^{23}$ W. Meyer, Int. J. Quantum Chem. Symp. 5, 341 (1971).
} 
${ }^{24}$ W. Meyer, J. Chem. Phys. 58, 1017 (1973)

${ }^{25}$ W. Meyer, Theor. Chim. Acta 35, 277 (1974).

${ }^{26}$ R. Ahlrichs, H. Lishka, V. Staemmler, and W. Kutzelnigg, J. Chem. Phys. 62, 1225 (1975).

${ }^{27}$ R. Ahlrichs, P. Scharf, and C. Ehrhardt, J. Chem. Phys. 82, 890 (1985).

${ }^{28}$ D. P. Chang and S. R. Langhoff, J. Chem. Phys. 84 (1986).

${ }^{29}$ J. P. Daudey, J. L. Heully, and J. P. Malrieu, J. Chem. Phys. 99, 1240 (1993).

${ }^{30}$ J. Rubio, A. Povill, F. Illas, and J. P. Malrieu, Chem. Phys. Lett. 200, 559 (1992).

${ }^{31}$ A. Povill, R. Caballol, J. Rubio, and J. P. Malrieu, Chem. Phys. Lett. 209, 126 (1993).

${ }^{32}$ R. Caballol and J. P. Malrieu, Chem. Phys. Lett. 188, 543 (1992).

${ }^{33}$ J. P. Malrieu, P. Durand, and J. P. Daudey, J. Phys. A 18, 809 (1985).

${ }^{34}$ P. Durand and J. P. Malrieu, in Ab initio Methods in Quantum Chemistry, edited by K. P. Lawley (Wiley, New York, 1986), Vol. 1, p. 321.

${ }^{35}$ J. Sánchez-Marín, D. Maynau, and J. P. Malrieu, Theor. Chim. Acta 87, 107 (1993)

${ }^{36}$ J. P. Malrieu, I. Nebot-Gil, and J. Sánchez-Marín, J. Chem. Phys. 100, 1440 (1994)

${ }^{37}$ Y. S. Lee, S. A. Kucharski, and R. J. Bartlett, J. Chem. Phys. 81, 5906 (1984).

${ }^{38}$ M. Urban, J. Noga, S. J. Cole, and R. J. Bartlett, J. Chem. Phys. 83, 4041 (1985).

${ }^{39}$ H. Feschbach, Ann. Rev. Nucl. Sci. 8, 49 (1958).

${ }^{40}$ H. Feschbach, Ann. Phys. (N.Y.) 5, 357 (1958).

${ }^{41}$ H. Feschbach, Ann. Phys. (N.Y.) 19, 287 (1962).

${ }^{42}$ P. O. Lowdin, J. Math. Phys. 3, 969 (1962).

${ }^{43}$ J. L. Heully and J. P. Malrieu, Chem. Phys. Lett. 199, 545 (1992).

${ }^{44}$ I. Lindgren and J. Morrison, Atomic Many-Body Theory (Springer, Berlin, 1986).

${ }^{45}$ R. A. Chiles and C. A. Dykstra, J. Chem. Phys. 74, 4544 (1981).

${ }^{46}$ N. C. Handy, J. A. Pople, M. Head-Gordon, K. Raghavachari, and G. W. Truks, Chem. Phys. Lett. 164, 185 (1989).
${ }^{47}$ I. Nebot-Gil, J. Sánchez-Marín, J. L. Heully, and J. P. Malrieu, Chem. Phys. Lett. 234, 45 (1995).

${ }^{48}$ S. A. Kucharski and R. J. Bartlett, J. Chem. Phys. 97, 4282 (1992).

${ }^{49}$ S. A. Kucharski and R. J. Bartlett, Chem. Phys. Lett. 206, 574 (1993).

${ }^{50}$ B. O. Roos and P. E. M. Siegbahn, in Methods of Electronic Structure Theory, edited by H. F. Schaefer III (Plenum, New York, 1977), p. 277.

${ }^{51}$ J. Olsen, B. O. Roos, P. Jørgensen, and H. J. Hensen, J. Chem. Phys. 89, 2185 (1988)

${ }^{52}$ J. P. Malrieu, J. P. Daudey, and R. Caballol, J. Chem. Phys. 101, 8908 (1994).

${ }^{53}$ M. B. Lepetit and J. P. Malrieu, J. Chem. Phys. 87, 5937 (1987).

${ }^{54}$ W. Kutzelnigg, Bad Honef Workshop on Coupled Cluster methods, Bad Honef (R.F.A.), October 1994.

${ }^{55}$ M. B. Lepetit and J. P. Malrieu, Chem. Phys. Lett. 208, 503 (1993).

${ }^{56}$ J. Meller, J. L. Heully, and J. P. Malrieu, Chem. Phys. Lett. 218, 276 (1994).

${ }^{57}$ D. Maynau and J. L. Heully, Chem. Phys. Lett. 211, 625 (1993).

${ }^{58}$ W. Diaz, I. Nebot-Gil, D. Maynau, and J. Sanchez-Marin (unpublished).

${ }^{59}$ G. E. Scuseria and T. J. Lee, J. Chem. Phys. 93, 5851 (1990).

${ }^{60}$ R. J. Harrison and N. C. Handy, Chem. Phys. Lett. 95, 386 (1983).

${ }^{61}$ P. Saxe, H. F. Schaefer III, and N. C. Handy, Chem. Phys. Lett. 79, 202 (1981).

${ }^{62}$ C. W. Bauschlicher, Jr. and P. R. Taylor, J. Chem. Phys. 85, 2779 (1986).

${ }^{63}$ K. Jankovski and J. Paldus, Int. J. Quantum Chem. 18, 1243 (1980).

${ }^{64}$ K. Jankovski, J. Paldus, and J. Wasilewski, J. Chem. Phys. 95, 3549 (1991).

${ }^{65}$ K. Jankovski, J. Paldus, I. Grabowski, and K. Kowalski, J. Chem. Phys. 97, 1600 (1992)

${ }^{66}$ P.-O. Widmark, P.-A. Malmqvist, and B. O. Roos, Theor. Chim. Acta 77, 291 (1990).

${ }^{67}$ S. Evangelisti, G. L. Bendazzoli, and L. Gagliardi, Chem. Phys. 185, 47 (1994).

${ }^{68}$ V. E. Bondybey, Chem. Phys. Lett. 109, 436 (1984). 